Check for updates

Cite this: RSC Adv., 2017, 7, 53172

Received 16th September 2017 Accepted 4th November 2017

DOI: $10.1039 / c 7 r a 10282 h$

rsc.li/rsc-advances

\section{para-Aramid fiber modified by melamine polyphosphate and its flame retardancy on thermoplastic polyurethane elastomer}

\begin{abstract}
Xilei Chen, (D) Wenduo Wang and Chuanmei Jiao*
With the increasing applications of thermoplastic polyurethane elastomer (TPU), the fire risk and smoke hazard caused by TPU have aroused wide attention. At the same time, with the promotion of aramid fiber in industry, the waste and pollution caused by aramid fiber is becoming worse. Herein, para-aramid fiber (AF) is recycled and modified by melamine polyphosphate (MP), and then applied as a flame retardant for TPU. The flame retardant and smoke suppression properties of the TPU composites are determined using the limiting oxygen index (LOI), cone calorimeter test (CCT), smoke density test (SDT) and thermogravimetric/Fourier transform infrared spectroscopy (TG-IR). The LOI test shows that the thermal stability of TPU is further improved by AF-MP. The CCT test shows that AF-MP has a better flame retardant and smoke suppression effect on TPU than pure AF. Remarkably, the pHRR and THR value for the sample with $1.00 \mathrm{wt} \%$ content of AF-MP decreased by $38.1 \%$ and $24.5 \%$ compared with that of the pure AF sample, where the residual mass of AF-MP was more than that of the AF sample. The SDT test shows that AF-MP has a better smoke suppression property in TPU than pure AF, particularly in the absence of a flame. The TG-IR test reveals that AF-MP further promotes the char forming process during combustion. Thus, this investigation presents great insight into the study of reusing fibers and efficient flame retardant polymers.
\end{abstract}

\section{Introduction}

TPU is widely used in many fields due to its excellent high tension, high tensile strength, toughness, resistance to aging properties, etc. ${ }^{1,2}$ However, similar to many other thermoplastics, due to its high flammability and the large amount of hightemperature toxic smoke released during its combustion, TPU has caused great pollution and harm to the environment. ${ }^{3-5}$ As a result, its applications in many fields are greatly limited. Therefore, the applications of effective flame retardants in TPU are quite important and urgent. ${ }^{6,7}$

Aramid fiber is a new type of high-tech synthetic fiber with excellent mechanical properties (ultra-high strength, high modulus, high temperature resistance, acid and alkali resistance, light weight, insulation, aging resistance, long life cycle, etc.), and with the gradual understanding of aramid fiber, it has been widely applied in many fields. ${ }^{8-10}$ At the same time, the waste and pollution caused by aramid fiber is becoming worse. Thus, the rational utilization and recycling of aramid fiber are necessary and significant. ${ }^{11}$

Aramid fiber is divided into meta-aramid fiber and paraaramid fiber. The main difference between these two types of

College of Environment and Safety Engineering, Qingdao University of Science and Technology, Qingdao, Shandong 266042, P. R. China. E-mail: jiaochm@qust.edu.cn; Fax: +86-532-84022703; Tel: +86-532-84022703 aramid fibers is the position where the amide bonds with the $\mathrm{C}$ atom on the benzene ring. ${ }^{12,13}$ para-Aramid fiber has better resistance to high temperature and other superior properties than that of meta-aramid fiber. Zhang Chong-geng et al. prepared a flame retardant using a halogen antimony flame retardant and aramid fiber to make an insulting layer in NBR, and the best formula ratio was obtained by testing different contents of halogen antimony flame retardant and aramid fiber. ${ }^{14}$ Zeng Cui-xia et al. made a blended flame retardant fabric by mixing aramid fiber and other natural fibers (cotton, wool, etc.) in a certain proportion..$^{15}$ Their research showed that the LOI increased and the mass loss of the blended flame retardant fabric decreased with an increase in the content of aramid fiber. In the field of polymer flame retardants, Chen Xi-lei et al. studied the fire safety of para-aramid fiber in TPU, and their results showed that para-aramid fiber could improve the flame retardancy and smoke suppression property of TPU considerably. ${ }^{16}$ This has opened a new field for the application of aramid fiber. However, there are some disadvantages with the use of aramid fiber. Aramid fiber is a material that is composed of highly oriented crystalline microregions with some defects and voids. Due to the steric effect of the large benzene ring in its molecular chain, it is difficult for the amide group to react with other atoms or groups. Thus, aramid fiber has poor adhesion with matrices because of its chemical inertia. ${ }^{17,18}$ In this case, some measures must be taken to reduce the surface orientation 
or increase the number of active groups on its surface. Only in this way can the bonding strength of the composite can be improved by forming reactive covalent bonds between its groups and the matrix. The methods for the modification of aramid fiber can be divided into groups: physical modification and chemical modification. Both methods achieve the same goal by forming some active center on the fiber surface and then enhancing the interaction between the fiber and matrix. ${ }^{19-21}$ In previous work, aramid fiber was modified by phosphoric acid and applied as a flame retardant in TPU, and the results showed that the modified aramid fiber had better flame retardancy that pure aramid fiber in TPU. ${ }^{\mathbf{1 1}}$

As an intumescent flame retardant (IFR), MP not only can be used alone as a flame retardant, but also be used in combination with other flame retardants. ${ }^{22-24}$ Particularly, MP shows better flame retardancy in the coating field than ammonium polyphosphate (APP). ${ }^{25,26}$ Mo Xian-zhong et al. studied the preparation of MP and its application in intumescent fire retardant coatings, and their results showed that MP could significantly improve the thermal insulation performance of fire retardant coatings. ${ }^{27}$ It was also reported that MP had been used as a reinforcing agent with glass fiber to investigate the flame retardancy in PA66, and the results showed that MP could apparently improve the flame retardancy of glass fiber in PA66. ${ }^{25,28,29}$ Li Hong-xia et al. studied the synergistic flame retardant effect between diethyl phosphate aluminium, MP and zinc borate in TPU, and their results showed that it could promote the char forming process and improve the flame retardancy of TPU. ${ }^{30}$ In a previous work, AF was modified by phosphoric acid and then added in TPU. Serious tests were carried out and the TPU composites containing modified AF showed better flame retardancy than the TPU with pure AF. ${ }^{\mathbf{1 1}}$ In this case, another idea can be proposed that there should be better flame retardancy in TPU when AF is modified by MP.

Herein, recycled para-aramid fiber (AF) is modified by MP, and TPU composites are prepared by mixing AF-MP into TPU via a melt blending method. A series of tests are conducted to study the flame retardant and smoke suppression effect of AF-MP on TPU. Subsequently, the flame retardant performance, smoke suppression effect and thermal stability of the samples are characterized via CCT, SDT and TG-IR respectively.

\section{Experimental}

\subsection{Materials}

TPU (9380A) was produced by Bayer, German. The basic properties of TPU are as follows: density: $1.11 \mathrm{~g} \mathrm{~cm}^{-3}$ (ISO1183); hardness: 82A (ISO868); tensile strength: $40 \mathrm{MPa}$ (ISO527-1, -3) and elongation at break: 500\% (ISO527-1, -3). para-Aramid fiber (XGPF1203) was supplied by Xiangu High-Tech. Co., Ltd. (Shenzhen, China). The basic properties of AF are as follows: density: $1.470 \mathrm{~g} \mathrm{~cm}^{-3}$ (ISO1183); short cut fiber length: $3 \mathrm{~mm}$; filament diameter: $12 \mu \mathrm{m}$; elastic modulus: 80-120 GPa (ISO527-2) and fracture strength: $19.4-21 \mathrm{cN}$ per dtex (ISO63831). Analytical reagent grade MP was obtained commercially.

\subsection{Sample preparation}

AF was added to washing liquid at a temperature of $95^{\circ} \mathrm{C}$ for $2 \mathrm{~h}$ to remove grease, dirt and other impurities from the fiber surface. The washing liquid contained a mass concentration of $2 \mathrm{~g} \mathrm{~L}^{-1}$ sodium carbonate and $1 \mathrm{~g} \mathrm{~L}^{-1}$ twelve sodium alkyl sulfonate, and the liquor ratio was $1: 50$. Finally, AF was washed with distilled water at a temperature of $50-60{ }^{\circ} \mathrm{C}$ to neutral and then dried in a drying oven.

The pre-treated AF was soaked in a saturated solution of MP with a $1: 20$ liquor ratio at $80{ }^{\circ} \mathrm{C}$, and kept stirring until the solution cooled to room temperature. Then, $\mathrm{AF}$ was filtered and dried in a drying oven at a temperature of $130{ }^{\circ} \mathrm{C}$ for $10 \mathrm{~min}$. The above processes were repeated 2 times. Finally, AF was washed with distilled water at a temperature of $50-60{ }^{\circ} \mathrm{C}$ to neutral, and AF-MP was obtained after drying in a drying oven.

The TPU composites were prepared via a melt blending method. TPU was dried in a vacuum oven at $80{ }^{\circ} \mathrm{C}$ for $12 \mathrm{~h}$ and then placed into a mixer and mixed at a rotation speed of $30 \mathrm{rpm}$ for $3 \mathrm{~min}$ at a temperature of $175 \pm 5{ }^{\circ} \mathrm{C}$. Then, AF-MP was added to the mixer and mixed for about $10 \mathrm{~min}$ to blend the polymer composites homogenously. Finally, the TPU composites were moulded with dimensions of $100 \times 100 \times 3 \mathrm{~mm}^{3}$ using a plate vulcanizer at a temperature of $175 \pm 5{ }^{\circ} \mathrm{C}$. The formulations of TPU composites are presented in Table 1.

\subsection{Measurements}

2.3.1. Scanning electron microscopy (SEM) and energy dispersive spectrometry (EDS). The surface characteristics of the samples were observed using a JEOL JSM 5900LV system for scanning electron microscopy (SEM) (JEOL, Japan) analysis at an accelerating voltage of $5 \mathrm{kV}$.

2.3.2. Limiting oxygen index (LOI). LOI was determined on an HC-2 oxygen index meter (Jiangning Analysis Instrument Company, China) according to the standard oxygen index test ASTM D2863. The dimensions of the samples were $100 \times 6.5 \times$ $3 \mathrm{~mm}^{3}$.

2.3.3. Cone calorimeter test (CCT). The flammability of the samples was measured using a PX-07-007 cone calorimeter device (Fire Testing Technology, UK). Samples with the dimensions of $100 \times 100 \times 3 \mathrm{~mm}^{3}$ were exposed to a radiant cone at a heat flux of $35 \mathrm{~kW} \mathrm{~m}^{-2}$.

2.3.4. Smoke density test (SDT). A smoke density test machine (JQMY-2, Jianqiao Co, China) was used to measure smoke characteristics according to ISO5659-2 (2006). Each specimen with dimensions of $75 \times 75 \times 2.5 \mathrm{~mm}^{3}$ was wrapped in aluminum foil and exposed horizontally to an external heat

Table 1 The formulations of TPU composites

\begin{tabular}{llll}
\hline Sample code & TPU $/ \%$ & AF $/ \%$ & AF-P/\% \\
\hline TPU & 100 & 0 & 0 \\
AF & 99.000 & 1.000 & 0 \\
AF-MP-1 & 99.750 & 0 & 0.250 \\
AF-MP-2 & 99.500 & 0 & 0.500 \\
AF-MP-3 & 99.000 & 0 & 1.000
\end{tabular}


flux of $25 \mathrm{~kW} \mathrm{~m}{ }^{-2}$ with or without the application of a pilot flame. Two tests were carried out for each sample.

2.3.5. Thermogravimetric analysis/infrared spectrometry (TG-IR). The thermal stability and thermal decomposition performance of the samples were tested on a thermogravimetric analyzer (DT-50) (Setaram Instrumentation Co., Led., France) at a heating rate of $20{ }^{\circ} \mathrm{C} \mathrm{min}^{-1}$ under a nitrogen flow of

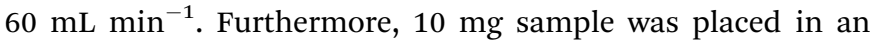
alumina crucible and the temperature ranged from $40{ }^{\circ} \mathrm{C}$ to $700{ }^{\circ} \mathrm{C}$.

Analysis of the components of the pyrolysis gas from the TG analyzer was performed using an FTIR spectrometer (170SX) (Shimadzu, Japan) in the wavenumber range of $4000-400 \mathrm{~cm}^{-1}$.

\section{Results and discussion}

\subsection{Scanning electron microscopy (SEM) and energy dispersive spectrometry (EDS)}

The SEM-EDS pictures of AF and AF-MP are shown in Fig. 1, which display the surface characteristics at the $10 \mu \mathrm{m}$ scale. Fig. 1(A) and (B) show the surface characteristics of AF and AFMP, and Fig. 1(C) and (D) show the elemental distribution in AF and AF-MP. It could be seen from Fig. 1(A) that there is no obvious twining between these fibers, and they are smooth and highly oriented and distributed regularly. In contrast, it could be seen in Fig. 1(B) that the fibers are rougher than pure AF, and they are curved and disorganized. This is because the physical characteristics of AF were changed by MP.
Moreover, it could be seen from Fig. 1(C) that $\mathrm{C}$ and $\mathrm{O}$ are the main elements in AF, and there is a little amount of $\mathrm{N}$ element. Compared with Fig. 1(C), it could be seen from Fig. 1(D) that P element and more $\mathrm{N}$ element exist in AF-MP. The weight ratio of $\mathrm{P}$ was $7.75 \mathrm{wt} \%$ and that of $\mathrm{N}$ was up to $5.81 \%$, which indicates that MP changed the chemical composition of AF, and the group containing $\mathrm{P}$ element and $\mathrm{N}$ element was coupled to the surface of AF successfully.

\subsection{Limiting oxygen index (LOI)}

LOI is defined as the minimum percentage of oxygen in an oxygen-nitrogen mixture that is just sufficient to sustain combustion of the sample after ignition. Higher LOI values make polymers more difficult to burn. The LOI test is widely used to evaluate the thermal stability properties of polymer materials, particularly for screening flame retardant formulations of polymers. ${ }^{2}$ The LOI results obtained from the TPU composites are presented in Fig. 2 . It could be seen obviously that the LOI of TPU is 23.5, and that of AF is 24.5, which is slightly higher than that of pure TPU. However, the LOIs of AFMP-1, AF-MP-2 and AF-MP-3 are 25.0, 26.5 and 27, respectively, which are much higher than those of TPU.

Thus, it could be concluded that AF modified by MP has better flame retardancy in TPU than AF. This is mainly because TPU and AF have the same amide bond and hydrogen bond, and AF can form a large conjugated aromatic structure with TPU. ${ }^{\mathbf{1 6}}$ It is known that aramid fiber has strong resistance to heat. Thus, the addition of AF increased the viscosity and strength of TPU. ${ }^{31}$
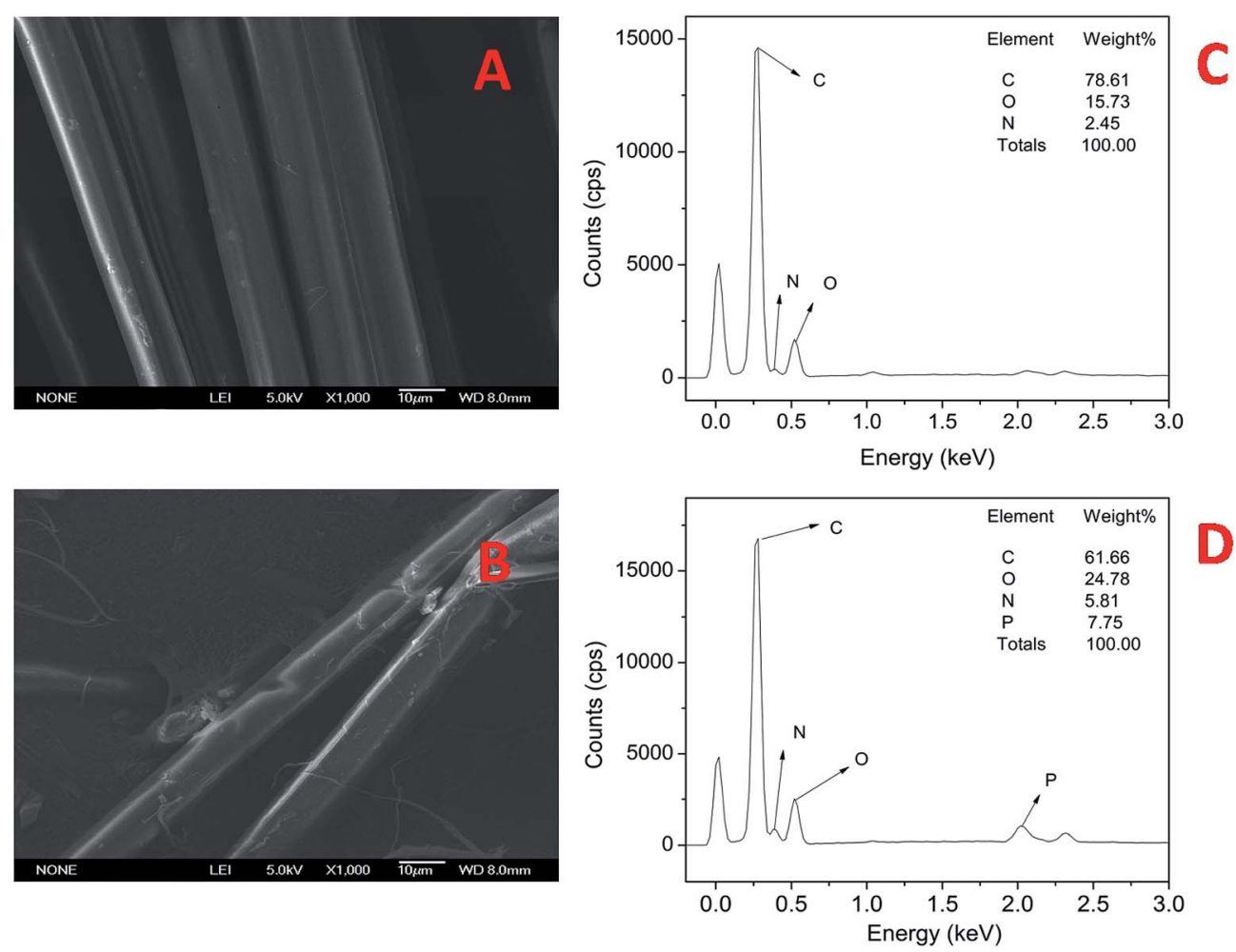

Fig. 1 SEM-EDS of AF (A), (C) and AF-MP (B), (D). 


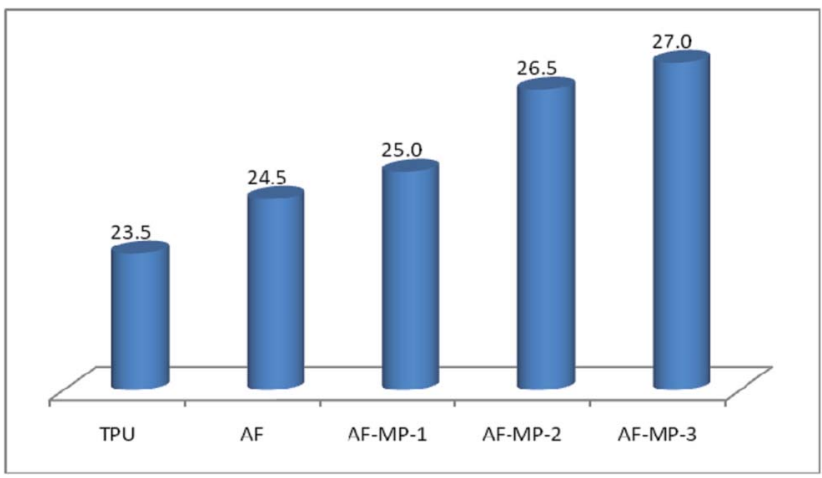

Fig. 2 LOI of the TPU composites.

In this case, when the TPU/AF composites were heated in CCT, the thermal stability of TPU increased, and as a result its LOI increased. Furthermore, as an important intumescent flame retardant (IFR), MP could hydrolyse at low temperature to release some polyphosphoric acid compounds and nonflammable gases, which could reduce the temperature of the polymer and dilute the oxygen concentration. In this way, the LOIs of the AF-MP samples were further improved.

\subsection{Cone calorimeter test (CCT)}

The cone calorimeter test (CCT) is based on the principle of oxygen consumption. It truly simulates the combustion of polymers in a real fire situation, and thus has great significance in the field of flame retardant materials. Some characteristic CCT data are shown in Table 2.

3.3.1. Heat release rate (HRR). The HRR curves of the TPU composites are shown in Fig. 3. It could be seen from Fig. 3 that the HRR of the AF-MP composites was much lower than that of pure TPU, and even lower than that of the AF sample, particularly the pHRR. The pHRR value of pure TPU was $1364 \mathrm{~kW} \mathrm{~m}^{-2}$, whereas that of the AF sample was $655 \mathrm{~kW} \mathrm{~m}^{-2}$, and the pHRR values of the AF-MP samples were as follows: $647 \mathrm{~kW} \mathrm{~m}^{-2}$, $520 \mathrm{~kW} \mathrm{~m}^{-2}$ and $447 \mathrm{~kW} \mathrm{~m}^{-2}$. Remarkably, the pHRR of AF-MP3 decreased by $67.2 \%$ compared with that of pure TPU and by $31.8 \%$ compared with that of the AF sample, which indicates that AF modified by MP has better flame-retardant ability in TPU than pure AF. This is mainly because AF could promote the char forming process, and furthermore, MP could improve the structure of the carbon layer. The formed carbon layer could be a barrier to stop heat and prevent the polymer from contacting air. As a result, less flammable particulates are released and the

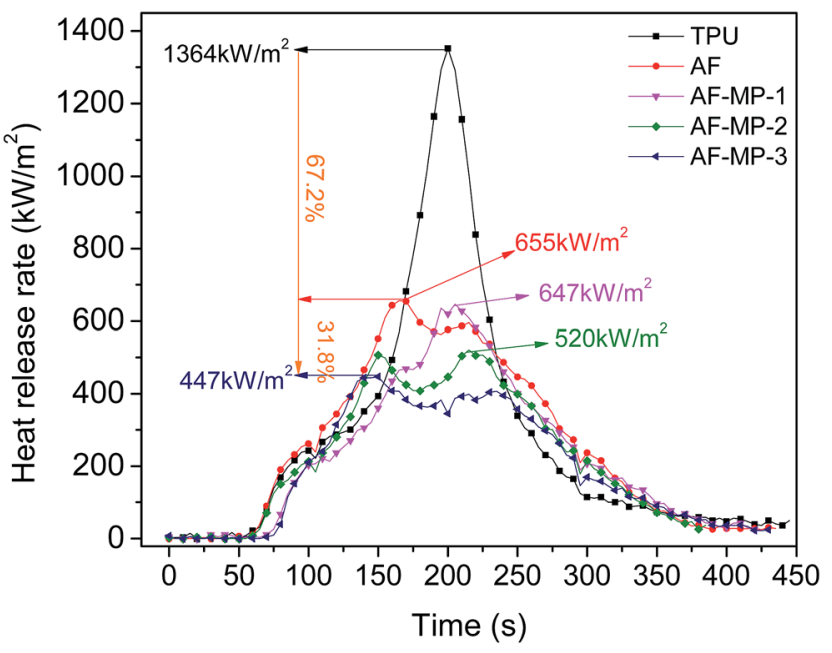

Fig. 3 HRR of the TPU composites at a flux of $35 \mathrm{~kW} \mathrm{~m} \mathrm{~m}^{-2}$.

HRR decreases. Another phenomenon that could be seen is that there was only one peak for pure TPU, whereas there were two peaks for the AF and AF-MP samples. This is due to the second decomposition of the char residue.

Thus, it could be concluded that AF improves the thermal stability of TPU and promotes the char forming process due to its large conjugated aromatic structure with TPU. When the samples were heated, the heat resistance of TPU was improved due to the addition of AF. Furthermore, in the process of the formation of the carbon layer, the char became more compact because of the uniform distribution of AF. As a widely applied IFR, MP further promoted the char forming process and improved the structure of the carbon layer. The $\mathrm{P}$ element formed some polyphosphoric acid compounds, which catalyzed the carbonization of TPU to generate a condensed carbon layer on the surface of TPU. The carbon layer acted as a barrier from heat, thus reducing the decomposition rate of the underlying materials to form flammable gases, resulting in a decrease in HRR. The N element formed some non-flammable gases, which could dilute the concentration of flammable gases and prevent the polymer from contacting air. Thus, the HRR of the AF-MP samples further decreased compared with that of AF sample, and the flame retardancy of TPU was further improved.

3.3.2. Total heat release (THR). The THR curves of the TPU composites are shown in Fig. 4 . It could be seen from Fig. 4 that the THR of the AF sample was lower than that of pure TPU at the end of the combustion. After AF-MP was added to TPU, the THR

Table 2 The characteristic data tested by a cone calorimeter

\begin{tabular}{|c|c|c|c|c|c|c|}
\hline TPU & 1364 & 6.2 & 121 & 872 & 0.089 & 1172 \\
\hline AF-MP-1 & 647 & 8.3 & 95 & 793 & 0.067 & 664 \\
\hline AF-MP-2 & 520 & 9.1 & 90 & 769 & 0.065 & 587 \\
\hline AF-MP-3 & 447 & 10.8 & 83 & 721 & 0.062 & 530 \\
\hline
\end{tabular}




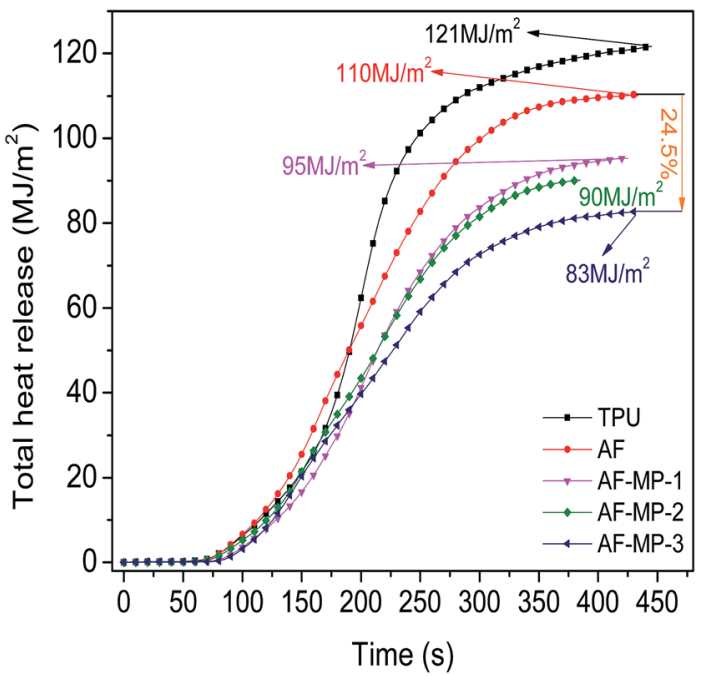

Fig. 4 THR of the TPU composites at a flux of $35 \mathrm{~kW} \mathrm{~m}^{-2}$.

was even lower than that of the AF sample. The THR of pure TPU at the end of the combustion was $121 \mathrm{MJ} \mathrm{m}^{-2}$; the HRR of the AF sample was $110 \mathrm{MJ} \mathrm{m}^{-2}$, whereas the HRR values of the AF-MP samples were $95 \mathrm{MJ} \mathrm{m}^{-2}, 90 \mathrm{MJ} \mathrm{m}^{-2}$, and $83 \mathrm{MJ} \mathrm{m}^{-2}$ respectively. With the increase in AF-MP, the THR values got lower and lower, where, the THR of AF-MP-3 decreased by $24.5 \%$ compared with that of the AF sample, remarkably. This could be explained by the fact that AF-MP improved the structure of the carbon layer and made the carbon layer more compact. The carbon layer acted as a barrier from heat and air, resulting in a lower THR, which is consistent with the HRR in Fig. 3. Above all, it also illustrates that the flame retardancy of TPU was further improved by AF-MP.

3.3.3. Smoke production rate (SPR). The SPR curves of the TPU composites are shown in Fig. 5. It can be seen that the SPR values of the sample containing AF and AF-MP are much lower than that of pure TPU overall, particularly for the peak value of SPR (pSPR). The pSPR of pure TPU was $0.089 \mathrm{~m}^{2} \mathrm{~s}^{-1}$, for the AF sample it was $0.073 \mathrm{~m}^{2} \mathrm{~s}^{-1}$, and for the AF-MP samples it was $0.067 \mathrm{~m}^{2} \mathrm{~s}^{-1}, 0.065 \mathrm{~m}^{2} \mathrm{~s}^{-1}$ and $0.062 \mathrm{~m}^{2} \mathrm{~s}^{-1}$. This is mainly because AF improved the resistance of TPU to heat due to its

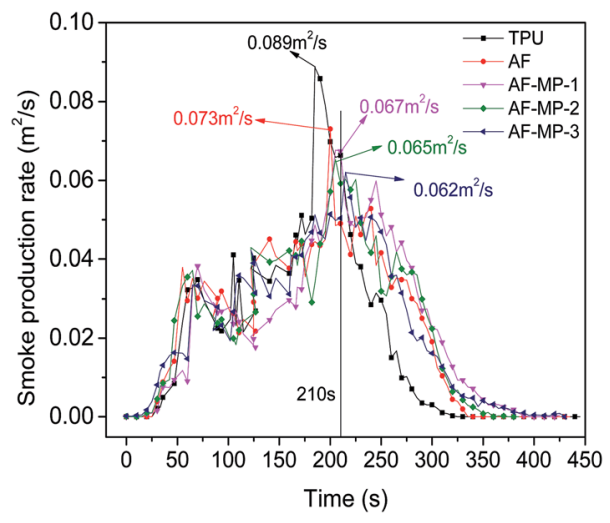

Fig. 5 SPR of the TPU composites at a flux of $35 \mathrm{~kW} \mathrm{~m}^{-2}$. large conjugated aromatic structure with TPU. Moreover, the existence of $\mathrm{AF}$ made the carbon layer more compact, which could separate heat or oxygen from the polymer and prevent smoke particles from being released into the fire. As a result, the SPR of the AF sample was lower than that of TPU. After the modification of AF by MP, MP could promote the char forming process and further improve the structure of the carbon layer, and the carbon layer acted as a barrier from heat and air. This reduced the decomposition rate of the underlying materials to form smoke particulates, resulting in a decrease in SPR, which is consistent with the HRR (Fig. 3) and THR (Fig. 4). Another special phenomenon that can be seen in Fig. 5 is that the SPR values of the $\mathrm{AF}$ and $\mathrm{AF}-\mathrm{MP}$ samples are higher than that of pure TPU after about $210 \mathrm{~s}$, which is a result of the second decomposition of the carbon layer. Overall, it can be concluded that AF-MP slows down the SPR, which is important for people in a fire accident.

3.3.4. Total smoke release (TSR). The TSR curves are presented in Fig. 6. It can be seen in Fig. 6 that the TSR of the AF sample is close to that of pure TPU, whereas the TSR of the AFMP samples is much lower than that of the pure TPU and $\mathrm{AF}$ samples. The TSR of pure TPU at the end of the combustion was $872 \mathrm{~m}^{2} \mathrm{~m}^{-2}$ and that of AF sample was $834 \mathrm{~m}^{2} \mathrm{~m}^{-2}$. In contrast, the TSR of the AF-MP samples was $793 \mathrm{~m}^{2} \mathrm{~m}^{-2}, 769 \mathrm{~m}^{2} \mathrm{~m}^{-2}$ and $721 \mathrm{~m}^{2} \mathrm{~m}^{-2}$, respectively. With an increase in the content of the AF-MP, the TSR values became lower. Remarkably, the TSR of AF-MP-3 decreased by $17.3 \%$ compared with that of pure TPU. This can be explained by the fact that AF-MP promotes the char forming process and further improves the structure of the carbon layer, and as a result, the carbon layer becomes more compact and difficult to decompose. The carbon layer acted as a barrier from heat and air, thus reducing the decomposition rate of the underlying materials to form smoke particulates, which resulted in a lower TSR. This is consistent with the SPR (Fig. 5). Thus, it can be concluded that MP efficiently improved the smoke suppression property of AF in TPU.

3.3.5. Smoke factor (SF). The SF curves of the TPU composites are presented in Fig. 7. It can be seen from Fig. 7 that the SF values of the samples containing $\mathrm{AF}$ are much lower

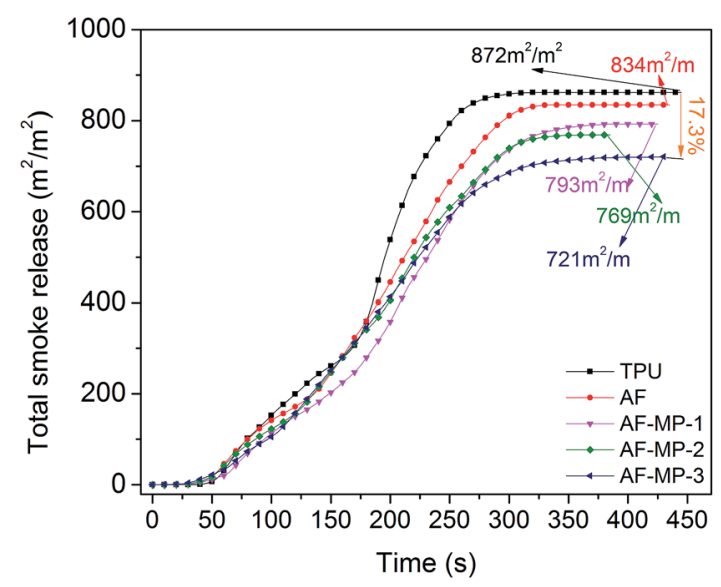

Fig. 6 TSR of the TPU composites at a flux of $35 \mathrm{~kW} \mathrm{~m}^{-2}$. 


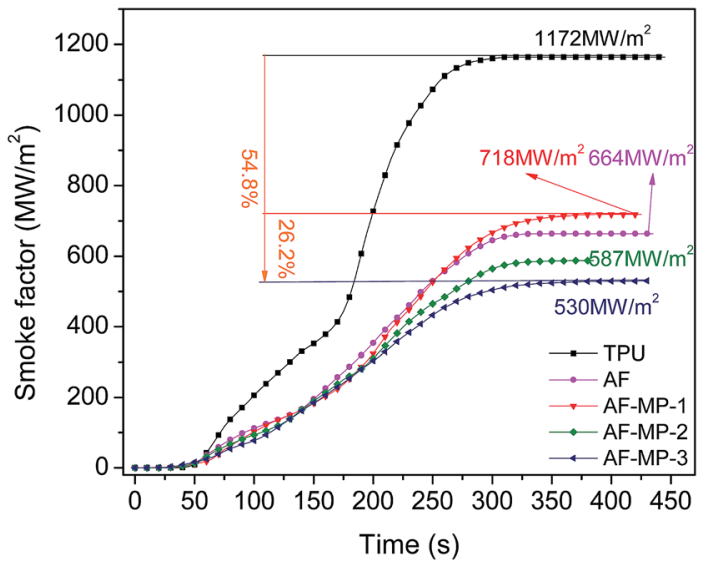

Fig. $7 \mathrm{SF}$ of the TPU composites at a flux of $35 \mathrm{~kW} \mathrm{~m}^{-2}$.

than that of pure TPU, and the SF values of the samples containing AF-MP are even lower than that of the AF sample. The SF value of pure TPU at the end of the combustion was about $1172 \mathrm{MW} \mathrm{m}^{-2}$ : for the AF sample it was about $718 \mathrm{MW} \mathrm{m}^{-2}$, and for the AF-MP samples it was $664 \mathrm{MW} \mathrm{m}^{-2}, 587 \mathrm{MW} \mathrm{m}^{-2}$ and $530 \mathrm{MW} \mathrm{m}^{-2}$, respectively. Remarkably, the SF value of AF-MP-3 decreased by $54.8 \%$ compared with that of pure TPU and $26.2 \%$ compared with that of the AF sample. Thus, it can be easily concluded that AF-MP further improves the flame retardancy and smoke suppression property of TPU compared to pure AF, which is consistent with the HRR (Fig. 3) and TSR (Fig. 6).

3.3.6. Mass. The residual mass curves of the TPU composites are presented in Fig. 8. It can be seen from Fig. 8 that the residual mass of the AF sample was slightly more than that of pure TPU, whereas the residual mass of the AF-MP samples was much more than that of pure TPU. The residual mass of pure TPU was $6.2 \%$, for the AF sample, it was $7.8 \%$, and for the AF-MP samples, it was $8.3 \%, 9.1 \%$ and $10.8 \%$.

Therefore, it is not difficult to draw the conclusion that AFMP plays a more important role in the char forming process than AF because AF-MP improves the structure of the carbon layer from TPU composites. As a result, the carbon layer is more

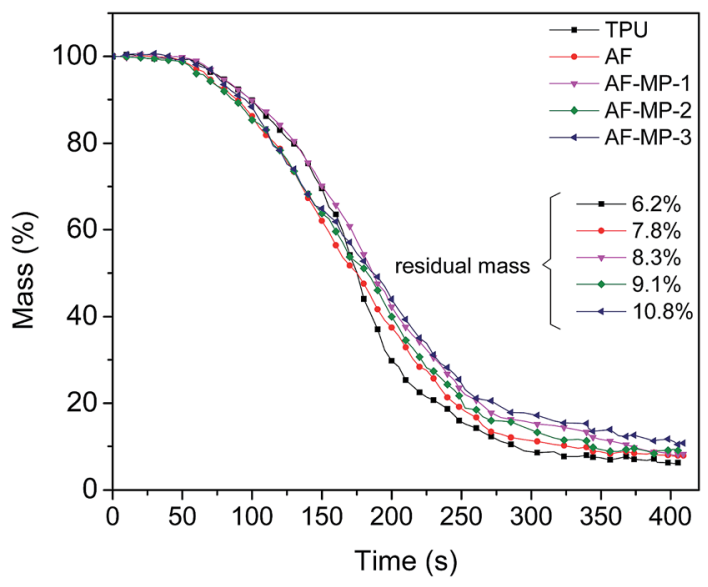

Fig. 8 Mass of the TPU composites at a flux of $35 \mathrm{~kW} \mathrm{~m}^{-2}$. compact to act as a barrier from heat and air, thus reducing the decomposition rate of the underlying materials to form flammable gases and smoke particulates, and enhancing the weight. The above results are in accordance with the HRR (Fig. 3) and TSR (Fig. 6) results.

3.3.7. Digital photographs of the residues. The digital photographs of the char residues after the cone calorimeter test are shown in Fig. 9. As can be seen in Fig. 9, the carbon layer of pure TPU at the end of the combustion was fragile and porous, which indicates that more polymer under the carbon layer decomposed, releasing more heat and smoke particulates. The carbon layer of the AF sample was more integrated and firmer, and there were no obvious pores on its surface, which indicates that $\mathrm{AF}$ improved the structure of the carbon layer. In contrast, in the samples with AF-MP, it can be seen that the carbon layer was not only integrated and compact, but also more expansive and firm. This result proves that AF-MP further improved the structure of the carbon layer. The formed carbon layer was more compact and expansive, and acted as an efficient barrier from heat and air, thus reducing the decomposition rate of underlying materials to form flammable gases and smoke particulates. This also resulted in a lower HRR and SPR, and the weight of the char residue was enhanced, which is consistent with the HRR (Fig. 3), SPR (Fig. 5) and mass (Fig. 8) results.

\subsection{Smoke density test (SDT)}

A smoke density test was carried out and the results are shown in Fig. 10. Fig. 10(A) presents the luminous flux curves of the TPU composites with flame in the SDT. It can be seen that the luminous flux of pure TPU rapidly decreased in the first $300 \mathrm{~s}$ and the lowest luminous flux value was obtained and it slightly increased later. However, the luminous flux of the AF sample decreased slightly slower than that of pure TPU in the first $300 \mathrm{~s}$. The difference between AF and the AF-MP samples was not obvious. When AF-MP was added to TPU, the luminous flux increased after reaching the lowest luminous flux value. This is mainly because MP had changed the composition of the smoke particulates, and when the combustion finished, the particulates sank and remained stable at the bottom.

Fig. 10(B) presents the luminous flux curves of the TPU composites without flame in the SDT. It can be seen that the luminous flux of TPU rapidly decreased in the first $500 \mathrm{~s}$ and the lowest luminous flux value $(2.7 \%)$ was obtained at the end of the combustion. The luminous flux of the AF sample was close to that of pure TPU in the initial $400 \mathrm{~s}$, and then decreased slowly and reached the lowest luminous flux value $(4.5 \%)$ at the end of the combustion, which was a little higher than that of pure TPU. This is mainly because AF promotes the char forming process due to its large conjugated aromatic structure with TPU. However, after AF-MP was added to TPU, the luminous flux values decreased more slowly, and with an increase in the content of AF-MP, the luminous flux value increased at the end of the combustion. Remarkably, in the case of AF-MP-3, the lowest luminous flux of $15.7 \%$ was achieved, which was much higher than that of the pure TPU and AF samples. This could be explained by the fact that as an IFR, MP further improves the 


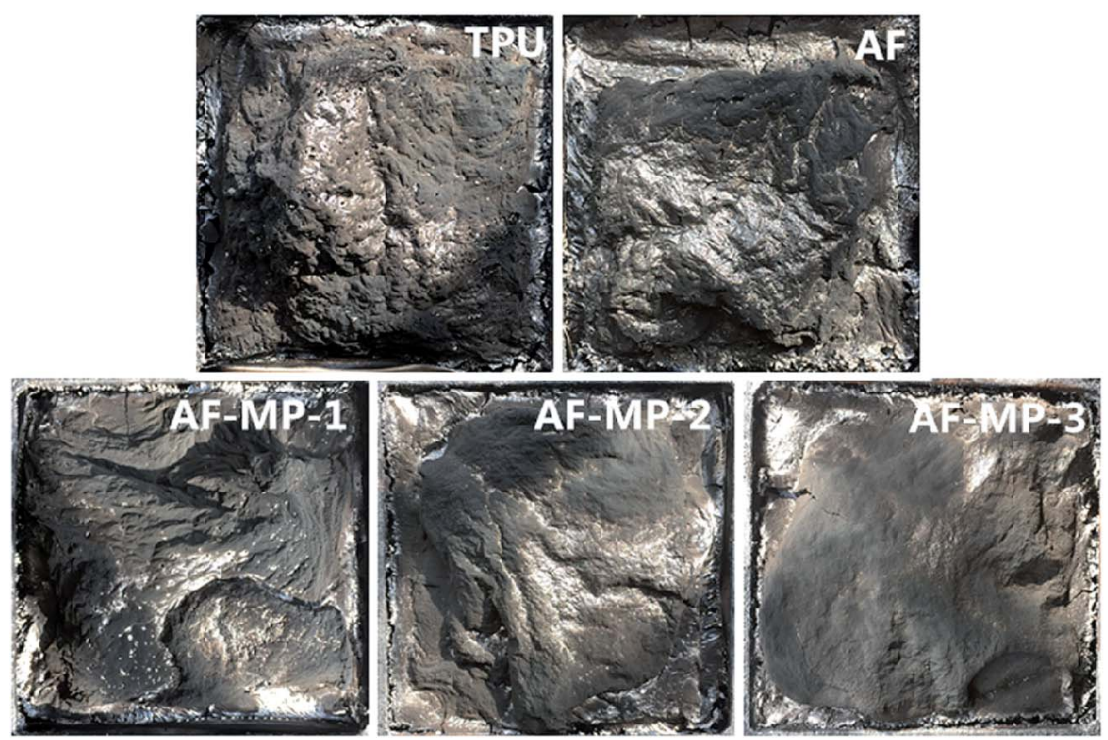

Fig. 9 Digital photographs of the residues after CCT.
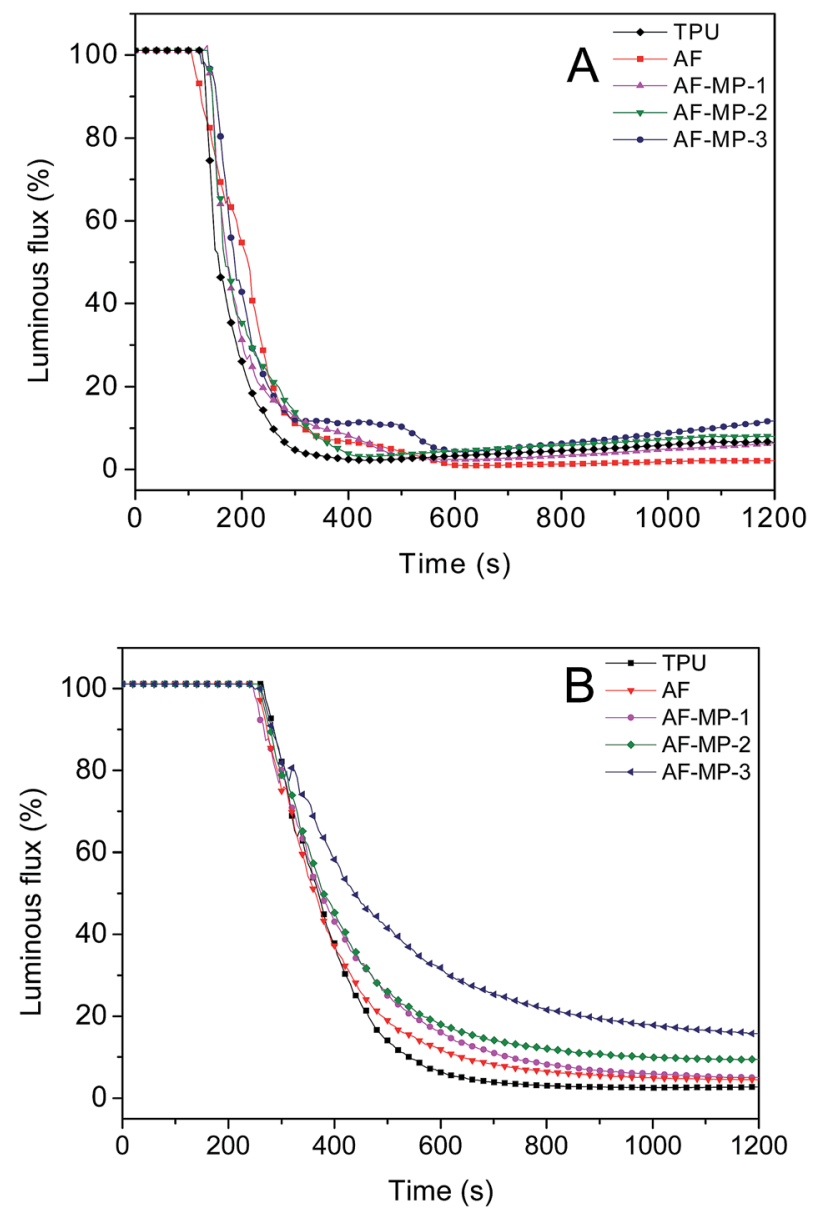

Fig. 10 Luminous flux curves of the TPU composites with flame (A) and without flame (B).

structure of the carbon layer. Furthermore, the carbon layer acted as a barrier from heat, which reduced the decomposition rate of the underlying materials to form smoke particulates, thus resulting in an increase in luminous flux. Thus, the luminous flux values were much higher than those of the AF sample. Therefore, it can be concluded that the smoke suppression property of TPU was further improved by AF-MP.

\subsection{Thermogravimetric analysis/infrared spectrometry (TG-IR)}

The infrared spectra from the pyrolysis of the TPU composites are presented in Fig. 11. The heating temperature range of the samples was $240-700{ }^{\circ} \mathrm{C}$ and the heating rate was $20 \mathrm{~K} \mathrm{~min}^{-1}$. It can be seen from the infrared spectra of pure TPU in Fig. 11(A) that there is a strong and broad absorption peak located at $2350 \mathrm{~cm}^{-1}$, which was formed by the cleavage of $\mathrm{CO}_{2}$. With an increase in temperature, the intensity of the absorption peak of $\mathrm{CO}_{2}$ increased gradually, reached the maximum at about $450{ }^{\circ} \mathrm{C}$, and then began to decline gradually. When the temperature increased to $520{ }^{\circ} \mathrm{C}$, the absorption peak of $\mathrm{CO}_{2}$ increased and remained stable, which indicates that $\mathrm{CO}_{2}$ was still being released. When the temperature increased to $340{ }^{\circ} \mathrm{C}$, a peak located at $1750 \mathrm{~cm}^{-1}$ appeared, which was attributed to the carbonyl group $(\mathrm{C}=\mathrm{O})$ in the TPU soft segment.

It can be seen from the infrared spectrum from the pyrolysis of the AF sample in Fig. 11(B) that the characteristic absorption peak of $\mathrm{CO}_{2}$ located at about $2350 \mathrm{~cm}^{-1}$ is much lower than that of TPU in the initial stage before $360{ }^{\circ} \mathrm{C}$. In addition, the characteristic absorption peak of $\mathrm{CO}_{2}$ for the $\mathrm{AF}$ sample was close to that of TPU between $360{ }^{\circ} \mathrm{C}$ and $520{ }^{\circ} \mathrm{C}$, but lower than that of pure TPU when the temperature increased from $520{ }^{\circ} \mathrm{C}$ to $700{ }^{\circ} \mathrm{C}$. In addition, the $\mathrm{C}=\mathrm{O}$ absorption peaks located at $1750 \mathrm{~cm}^{-1}$ for the AF sample were slightly higher than those of pure TPU.

It can be seen from the infrared spectrum from the pyrolysis of the AF-MP-3 sample in Fig. 11(C) that the characteristic absorption peak of $\mathrm{CO}_{2}$ located at about $2350 \mathrm{~cm}^{-1}$ was much lower than that of pure TPU before $360^{\circ} \mathrm{C}$, which then increased 

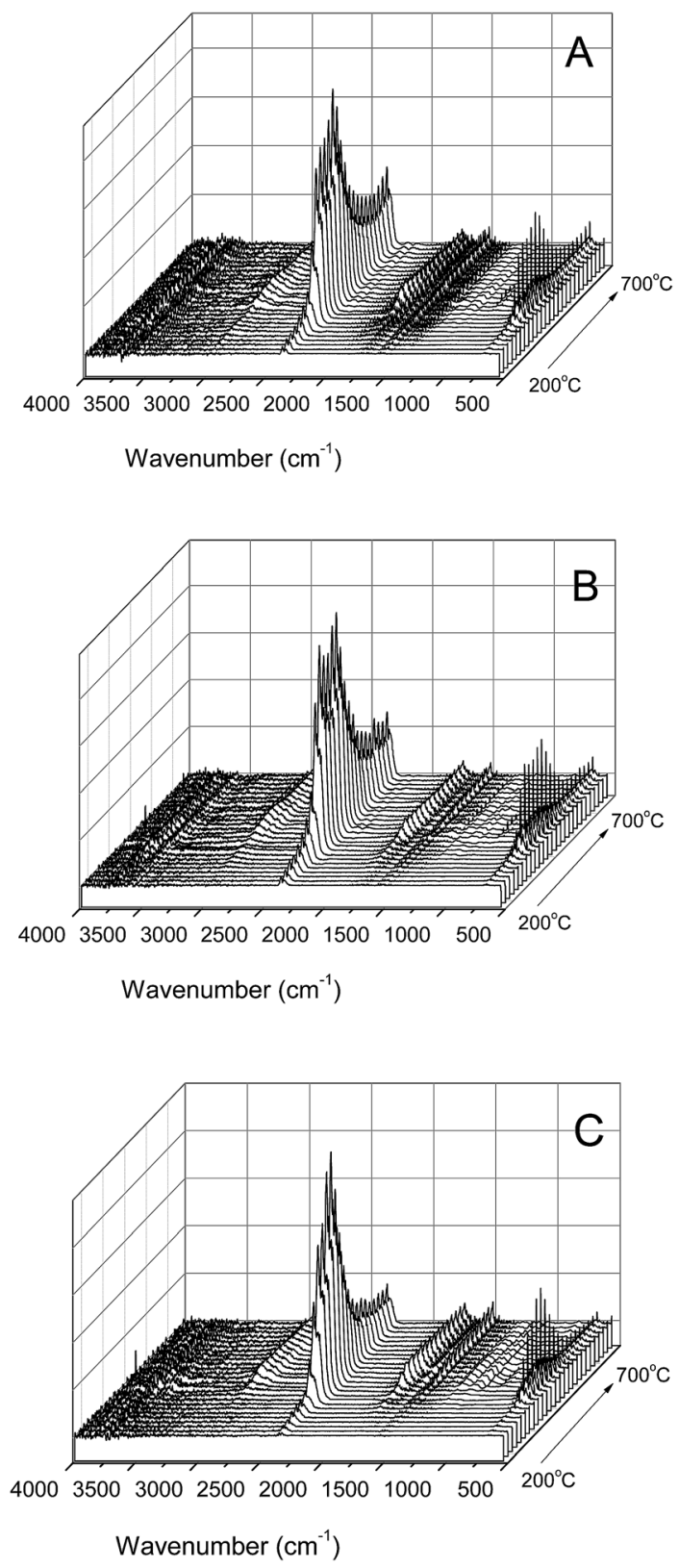

Fig. 11 TG-IR curves of TPU (A), AF (B) and AF-MP-3 (C) samples.

and became close to that of pure TPU. When the temperature reached about $420{ }^{\circ} \mathrm{C}$ the peak started to decrease rapidly and then remained stable, which was much lower than that of the pure TPU and AF samples. Moreover, the $\mathrm{C}=\mathrm{O}$ absorption peaks located at $1750 \mathrm{~cm}^{-1}$ for the AF-P-3 sample were slightly higher than that of pure TPU and AF.

Therefore, it could be easily concluded that the thermal stability of TPU increased by the addition of AF, and thus the characteristic absorption peak of $\mathrm{CO}_{2}$ in $\mathrm{AF}$ was much lower than that of pure TPU at a lower temperature. In addition, with time, the $\mathrm{CO}_{2}$ absorption peak in $\mathrm{AF}$ was lower than that of pure TPU at about $520^{\circ} \mathrm{C}$, which is because AF promoted the char forming process due to its large conjugated aromatic structure with TPU. However, in AF-MP-3, as an IFR, MP further promoted the char forming process and improved the structure of the carbon layer. Furthermore, MP could form polyphosphoric acid compounds, which catalyze the carbonization of TPU to generate a condensed carbon layer on the surface of TPU. The formed carbon layer acted as a barrier from heat and air, reducing the decomposition rate of the underlying materials to form flammable gases and smoke particulates, and as a result less $\mathrm{CO}_{2}$ was released. This is consistent with the results obtained by CCT.

\section{Conclusion}

Since the fire hazard caused by TPU and the pollution caused by aramid fiber are becoming more serious, some measures must be taken to alleviate this situation. Herein, research on the flame retardancy of aramid fiber modified by MP in TPU was carried out. The results show that AF-MP has better flame retardancy and smoke suppression property on TPU than pure AF. Therefore, we provide a new strategy and method to reduce the fire hazard of TPU and the pollution of aramid fiber. This is very significant for the reuse of abandoned resources and the development of green flame retardant polymers.

\section{Conflicts of interest}

There are no conflicts to declare.

\section{Acknowledgements}

The authors gratefully acknowledge the National Natural Science Foundation of China (No. 51776101, 51206084), the Major Special Projects of Science and Technology from Shandong Province (2015ZDZX11011), the Natural Science Foundation of Shandong Province (ZR2017MB016), and the Project of the State Administration of Work Safety (shandong-00392017AQ).

\section{References}

1 G. Wypych, Handbook of Polymers, 2nd edn, 2016, pp. 686689.

2 C. Jiao, H. Wang, S. Li and X. Chen, J. Hazard. Mater., 2017, 332, 176-184.

3 D. Tabuani, F. Bellucci, A. Terenzi and G. Camino, Polym. Degrad. Stab., 2012, 97, 2594-2601.

4 A. Toldy, G. Harakály, B. Szolnoki, E. Zimonyi and G. Marosi, Polym. Degrad. Stab., 2012, 97, 2524-2530.

5 X. Chen, W. Wang and C. Jiao, RSC Adv., 2016, 6, 9227692284.

6 U. Almeida Pinto, L. L. Y. Visconte, J. Gallo and R. C. R. Nunes, Polym. Degrad. Stab., 2000, 69, 257-260.

7 H. Li, N. Ning, L. Zhang, Y. Wang, W. Liang and M. Tian, Polym. Degrad. Stab., 2014, 105, 86-95.

8 K. Akato, and G. Bhat, Structure and Properties of HighPerformance Fibers, 2017, pp. 245-266.

9 J. K. Fink, High Performance Polymers, 2nd edn, 2014, pp. 301-320. 
10 Z. Li, X. Cheng, S. He, X. Shi, L. Gong and H. Zhang, Composites, Part A, 2016, 84, 316-325.

11 X. Chen, W. Wang and C. Jiao, J. Hazard. Mater., 2017, 331, 257-264.

12 Z. Sun, S. Shi, X. Hu, X. Guo, J. Chen and H. Chen, Composites, Part B, 2015, 77, 38-45.

13 B. L. Deopura and N. V. Padaki, in Textiles and Fashion, 2015, pp. 7-114.

14 C. g. Zhang, H. l. Wang, Q. LI, L. Cong and X. h. Zhang, J. Solid Rocket Technol., 2008, 31, 635-637.

15 C. Zeng, Shanghai Textile Science \& Technology, 2008, 37, 4748.

16 X. Chen, W. Wang, S. Li and C. Jiao, J. Hazard. Mater., 2017, 324, 789-796.

17 J. Chen, Y. Zhu, Q. Ni, Y. Fu and X. Fu, Appl. Surf. Sci., 2014, 321, 103-108.

18 R. Gu, J. Yu, C. Hu, L. Chen, J. Zhu and Z. Hu, Appl. Surf. Sci., 2012, 258, 10168-10174.

19 R. Sa, Y. Yan, Z. Wei, L. Zhang, W. Wang and M. Tian, ACS Appl. Mater. Interfaces, 2014, 6, 21730-21738.

20 C. X. Wang, M. Du, J. C. Lv, Q. Q. Zhou, Y. Ren, G. L. Liu, D. W. Gao and L. M. Jin, Appl. Surf. Sci., 2015, 349, 333-342.
21 M. Xi, Y.-L. Li, S.-y. Shang, D.-H. Li, Y.-X. Yin and X.-Y. Dai, Surf. Coat. Technol., 2008, 202, 6029-6033.

22 X. Bai, G. Geng, S. Hu and A. Zhang, Paint Coat. Ind., 2012, 42(6), 64-66.

23 C. Wang, J. Li and P. Ding, J. Anal. Appl. Pyrolysis, 2016, 119, 139-146.

24 S. Zhou, Z. Wang, Z. Gui and Y. Hu, Fire Mater., 2008, 32, 307-319.

25 U. Braun, B. Schartel, M. A. Fichera and C. Jäger, Polym. Degrad. Stab., 2007, 92, 1528-1545.

26 Y. Chen and Q. Wang, Polym. Adv. Technol., 2007, 18, 587600.

27 X. Mo, Z. Liao and C. Gan, J. Funct. Mater., 2004, 35, 17251727.

28 Z. Wang, Y. Liu and Q. Wang, Eng. Plast. Appl., 2007, 35(3), 47.

29 S. V. Levchik, A. I. Balabanovich, G. F. Levchik and L. Costa, Fire Mater., 1997, 21, 75-83.

30 H. Li, L. Zhang, Y. Wang, W. Liang and M. Tian, China Plast. Ind., 2014, 42(4), 76-80.

31 L. Loureiro, V. Henrique Carvalho and S. H. Prado Bettini, Polym. Test., 2016, 56, 124-130. 